\title{
Curso de extensão universitária para professores de Ciências da Natureza sobre o tema "Terra e universo"
}

\author{
University eXtension cOURSE fOR teachers of Natural Sciences on the thematic unit "Earth and UNIVERSE"
}

\author{
Elias Araujo Bressane ${ }^{1}$, Maria Cristina Motta de Toledo², Celso Dal Ré Carneiro $0^{3,4}$ \\ 1 - Doutorando no Progr. Pós-Graduação em Ensino e História de Ciências da Terra, Instituto de Geociências, Universidade Estadual de Campinas, Caixa Postal \\ 6152, 13083-970 CampINAS, SP, BRASIL. \\ 2 - Profa. Titular da Esc. Ciências, Artes e Humanidades usp, e Professora Permanente do Progr. Pós-Graduação em Ensino e História de Clências da Terra, \\ Instituto de Geociências, Universidade Estadual de Campinas, Campinas, SP, Brasil. \\ 3 - Progr. Pós-Graduação em Ensino e História de Clências da Terra, Instituto de Geociências, Universidade Estadual de Campinas, Caixa Postal 6152, 13083- \\ 970 Campinas, SP, BRAsLl. 4Bolsista do CNPQ. \\ E-MALLS: ElASARAUJO@IGE.uNICAMP.BR, MCRISTOL@USP.BR, CEDREC@IGE.UNICAMP.BR
}

Abstract: The National Common Curricular Base approved by the Ministry of Education presents themes of Earth Sciences that must be developed in all the years of elementary education. However, studies have identified insufficient content of Earth Science in the curricula of the initial formation of Pedagogues, which usually teach from the $1^{\text {st }}$ to the $5^{\text {th }}$ years of elementary education, and by teachers of Natural Sciences, who teach from the $6^{\text {th }}$ to the $9^{\text {th }}$ years of the same cycle of basic education. Thus, it is necessary to complement the initial teacher training. An alternative is the extension courses, which would strengthen the academic knowledge of teachers. This paper aims to describe a proposal for a course of improvement, classified as semi-presencial university extension, focusing on fundamental themes of Earth Sciences. The course is designed for teachers who teach Natural Sciences in basic schools. As long as it is a pilot project to be developed at the Institute of Geosciences of Unicamp, under the administration of the School of Extension also of Unicamp, quantitative and qualitative research will be done to promote adjustments that may lead to a final proposal, which may be offered periodically. The pedagogical project will be made available to other higher education institutions that may be interested.

\section{Introdução}

O texto oficial da Base Nacional Comum Curricular (BNCC) foi homologado pelo Ministério da Educação no final do ano de 2017 e, por seu conteúdo, reforça os objetivos do trabalho aqui apresentado. Este artigo descreve uma proposta de curso de extensão universitária, na modalidade de aperfeiçoamento, como projeto-piloto, que objetiva valorizar assuntos básicos da Geologia, conceitos e dinâmicas do ensino de Ciências da Terra, além de outras abordagens descritas na unidade temática "Terra e Universo" da disciplina de Ciências da Natureza. O curso de extensão integra um conjunto de atividades de projeto de pesquisa de doutorado em andamento, sendo destinado a duas categorias de professores do ensino fundamental: os responsáveis pela docência nos anos iniciais e pela área de Ciências da Natureza dos anos finais do ensino fundamental.
Manuscrito:

Recebido: Quadrennial Conference of the International Geoscience Education Organization

Aceito: 13/03/2019

Citação: Bressane, E. A., Motta de Toledo, M. C., Carneiro, C. D. R. 2019. Curso de extensão universitária para professores de Ciências da Natureza sobre o tema "Terra e universo". Terræ Didatica, 15, 1-8, e19021. doi: 10.20396/td.v15i0.8654875

Palavras-chave: Base Nacional Comum Curricular, formação de professores, Ciências da Terra, ensino fundamental, Terra e Universo.

Linha temática: Geociências e Ciências Naturais para Educação Básica.

Geralmente, os cursos de Pedagogia que habilitam professores para atuar nos anos iniciais do ensino fundamental não oferecem disciplinas específicas de Ciências da Terra na grade curricular. Em algumas universidades há disciplinas que eventualmente se aproximam, como Educação Ambiental. Contudo, é raro haver disciplinas como Geologia Geral ou algo semelhante. Já as Licenciaturas em Biologia, que habilitam professores para atuar no ensino de Ciências da Natureza nos anos finais do mesmo ensino fundamental, possuem, por vezes, na grade curricular, uma disciplina de Geologia e outra de Paleontologia, ou, em alguns casos, uma única disciplina para formar alunos nas duas áreas do conhecimento científico.

Assim, parece oportuno e necessário organizar cursos de extensão universitária para oferecer aperfeiçoamento em Ciências da Terra a professores que lecionam Ciências da Natureza no ensino 
fundamental, ainda mais quando a BNCC recém homologada valoriza a interdisciplinaridade e a presença do conhecimento em Ciências da Terra para a formação escolar.

Para favorecer a participação dos interessados, que exercem suas atividades profissionais em horários diversificados, o curso de aperfeiçoamento seria organizado no formato semipresencial, utilizando a plataforma Moodle e seguindo as normas que regem cursos de extensão na universidade. Mais adiante são apresentadas informações sobre carga horária mínima e disciplinas previstas.

A pesquisa envolve avaliação dos resultados obtidos pelos concluintes do curso, mediante verificação das percepções apresentadas por todos os envolvidos, como coordenadores do curso, professores, alunos e outros, com o propósito de realizar correções e ajustes. O curso deverá atender aos princípios indissociáveis da universidade, definidos no artigo 207 da Constituição Federal (Brasil 1988), que são a pesquisa, o ensino e a extensão. Com base na experiência, serão realizadas propostas fundamentadas em estudos quantitativos e qualitativos, incluindo-se a demanda de interessados, para torná-lo um curso periódico de extensão universitária a ser oferecido pela Unicamp ou outras universidades interessadas.

\section{Objetivos}

O objetivo principal desta fase da pesquisa é desenvolver um curso de extensão universitária para professores de Ciências da Natureza, como projeto-piloto, que apresente propostas de valorização das Ciências da Terra, de acordo com as áreas de conhecimento da unidade temática Terra e Universo, propostas pela nova BNCC para o ensino fundamental, com a finalidade de oferecer e discutir com esses professores novas técnicas e conhecimentos em Ciências da Terra.

Ao final dos trabalhos, seriam realizadas análises dos resultados obtidos no curso, com o uso de questionários que possibilitem a obtenção de dados quantitativos e qualitativos, além da consideração das observações efetuadas durante todo o curso. Assim, seria possível reajustar o curso de aperfeiçoamento para um modelo que atendesse aos interesses da educação básica, da Universidade, do Instituto que o desenvolver e, especialmente, do público alvo desse trabalho, viabilizando o objetivo primordial desta proposta, que é a valorização das Ciências da Terra na disciplina de Ciências da Natureza.

\section{Métodos}

Para alcançar o objetivo proposto, o trabalho de pesquisa deverá ser organizado em determinadas fases. Inicialmente, será analisado o curso desenvolvido pelo IG em 2016, sob amparo técnico-administrativo da Escola de Extensão da Unicamp (Extecamp), identificado como "GEO-032 Geociências para Educação Básica" e destinado a professores da rede oficial de ensino. Os resultados obtidos na análise servirão de base para a formulação de um novo curso para professores de Ciências da Natureza, de acordo com as áreas de conhecimento em “Terra e Universo" apresentadas pela BNCC do ensino fundamental.

Deverão ser realizadas consultas junto à Secretaria de Educação da Prefeitura Municipal de Campinas, para obtenção de dados referentes ao público-alvo. Depois da proposta do curso ser elaborada sob a coordenação do IG, será apresentada à Extecamp e o curso será desenvolvido com a colaboração de alunos dos programas de pós-graduação mantidos pelo IG.

Os conteúdos e a preparação do curso de extensão dependem de aprofundados estudos sobre as áreas de conhecimento em Terra e Universo, propostas pela BNCC para as Ciências da Natureza do ensino fundamental. Os temas identificados de Astronomia, Meteorologia e Geologia serão organizados de forma interdisciplinar. Depois de desenvolvido o curso, será necessária a avaliação de resultados junto aos alunos. Por fim, o próprio curso será avaliado pelos seus integrantes, para os reajustes indispensáveis.

\subsection{Forma de levantamento, interpretação e análise dos dados}

Conforme já citado, serão realizadas observações e discussões ao longo do curso, que poderão subsidiar reflexões e eventualmente propostas de ajustes nas várias etapas. Também, no transcorrer do curso, serão desenvolvidas pesquisas quantitativas e qualitativas, por meio de questionários e entrevistas envolvendo os discentes, docentes e coordenação do curso sob proposta.

A pesquisa quantitativa terá por objetivo o levantamento de referências sobre a demanda de vagas e interessados, o perfil dos participantes, a estrutura do curso e outras informações obtidas por meio da coleta de dados estatísticos. Por sua vez, a pesquisa qualitativa abordará as motivações que 
levaram os interessados a participarem do curso, suas percepções sobre a importância das abordagens e do aprofundamento nos conhecimentos dos temas propostos na BNCC e relacionados às Ciências da Terra no ensino fundamental, a construção de novas práticas de ensino que possivelmente agregaram durante as dinâmicas do curso e, enfim, as demais evoluções às quais foram conduzidos durante a integração com os demais profissionais da educação e as propostas trabalhadas durante o curso de extensão.

Os dados quantitativos levantados e as informações qualitativas obtidas pela investigação serão mensurados e analisados e o conjunto de seus elementos poderá contribuir para subsidiar estudos que conduzam à redefinição de conceitos e parâmetros referentes ao curso de extensão em estudo, tornando possível a reformulação de planejamentos destinados à organização de um modelo de curso que se relacione com as realidades do ensino fundamental e suas características e necessidades estruturais, sociais e educacionais. Nestas características e necessidades, estão incluídas a formação para a cidadania e a educação ambiental, que conduzem para comportamentos que se aproximem da sustentabilidade e outras indagações relacionadas à natureza que integramos.

Como todo projeto que objetiva a discussão e construção de conhecimentos, esse não findará com a proposta inicial ou depois de eventual reformulação, porque sempre existirá a necessidade de ajustes que acompanhem a evolução da demanda dos diversos ciclos da educação básica.

\section{0 s antecedentes da Base Nacional Comum Curricular}

De acordo com o artigo 214 da Constituição Federal (Brasil 1988), deve ser estabelecido um Plano Nacional de Educação (PNE), de duração decenal, com o objetivo de articular o Sistema Nacional de Educação em regime de colaboração, e definir diretrizes, objetivos, metas e estratégias de implementação para assegurar a manutenção e desenvolvimento do ensino em seus diversos níveis, etapas e modalidades por meio de ações integradas dos poderes públicos das diferentes esferas federativas que conduzam a: (a) erradicação do analfabetismo; (b) universalização do atendimento escolar; (c) melhoria da qualidade do ensino; (d) formação para o trabalho; (e) promoção humanística, científica e tecnológica do país; (f) estabelecimento de meta de aplicação de recursos públicos em educação como proporção do produto interno bruto.

Com base nessas metas, em 2014, a Lei $n^{\circ}$ 13.005 (Brasil 2014) aprovou o citado PNE, que reitera a necessidade de estabelecer e implantar, mediante pacto federativo (União, Estados, Distrito Federal e Municípios), diretrizes pedagógicas para a educação básica e a base nacional comum dos currículos, com direitos e objetivos de aprendizagem e desenvolvimento dos alunos para cada ano dos ensinos fundamental e médio, respeitadas as diversidades regional, estadual e local (Brasil 2014). Nesse sentido, o PNE reitera a importância de uma base nacional comum curricular para o Brasil, com o foco na aprendizagem como estratégia para fomentar a qualidade da educação básica em todas as etapas e modalidades.

Assim, segundo informações constantes na página eletrônica do Ministério da Educação, foram apresentadas à sociedade versões de uma BNCC para a educação básica, diferenciada para os ensinos fundamental e médio.

Trata-se de um documento de caráter normativo que define o conjunto orgânico e progressivo de aprendizagens essenciais que todos os alunos devem desenvolver ao longo das etapas e modalidades da educação básica.

No final do ano de 2017, a BNCC para o ensino fundamental (Brasil 2017), compreendido do $1^{\circ}$ ao $9^{\circ}$ ano da educação básica, ou dos 6 aos 14 anos de idade escolar, foi homologada pelo Ministério da Educação.

Conforme definido pela Lei de Diretrizes e Bases da Educação Nacional (LDB), a BNCC deve ser desenvolvida para ser aplicada à educação escolar e indicar conhecimentos e competências que se espera que todos os estudantes desenvolvam ao longo da escolaridade (Brasil 1996).

\subsection{A Base Nacional Comum Curricular}

O ensino fundamental, período escolar de interesse desta pesquisa, está organizado na BNCC em quatro áreas do conhecimento: Linguagens (Língua Portuguesa, Artes, Educação Física e Língua Inglesa); Matemática (Matemática); Ciências da Natureza (Ciências); Ciências Humanas (Geografia e História).

A área de conhecimento de Ciências da Natureza é apresentada em três unidades temáticas: Matéria e Energia; Vida e Evolução; Terra e Uni- 
verso. A unidade temática Terra e Universo abrange temas de Ciências da Terra que possivelmente não são tratados na formação de professores para os anos iniciais do ensino fundamental e de Ciências da Natureza dos anos finais da mesma fase da educação básica, principalmente quando nos referimos, respectivamente, aos cursos de Licenciaturas em Pedagogia e em Ciências Biológicas, titulações comumente referenciadas em editais de concurso para admissão desses professores.

Os objetos de conhecimento da unidade temática Terra e Universo, propostos pela BNCC, são:

- $\mathbf{1}^{\mathbf{o}}$ ano: escalas de tempo;

- $\quad 2^{\mathbf{O}}$ ano: movimento aparente do Sol no céu; o Sol como fonte de luz e calor;

- $\quad 3^{\mathbf{0}}$ ano: características da Terra; observação do céu; usos do solo;

- $4^{\mathrm{o}}$ ano: pontos cardeais; calendários, fenômenos cíclicos e cultura;

- $\quad \mathbf{5}^{\mathbf{o}}$ ano: constelações e mapas celestes; movimento de rotação da Terra; periodicidade das fases da Lua; instrumentos ópticos;

- $6^{\mathbf{0}}$ ano: forma, estrutura e movimentos da Terra;

- $\quad 7^{\mathbf{0}}$ ano: composição do ar; efeito estufa; camada de ozônio; fenômenos naturais (vulcões, terremotos e tsunamis); placas tectônicas e deriva continental;

- $\mathbf{8}^{\mathbf{o}}$ ano: sistema Sol, Terra e Lua; clima; e

- $\quad 9^{0}$ ano: composição, estrutura e localização do Sistema Solar no Universo; astronomia e cultura; vida humana fora da Terra; ordem de grandeza astronômica; evolução estelar.

Esses objetos de conhecimento, com rico conteúdo em Ciências da Terra, são detalhados pela BNCC em habilidades específicas.

Durante a fase de consultas públicas para a consolidação da BNCC, Soares (2016) apresentou ao Ministério da Educação propostas sobre os componentes curriculares de Terra e Universo, enfatizando a ideia de conteúdos próximos da realidade do cotidiano social. Para o autor, a educação em Ciências da Terra deve aproximar os estudantes ao meio em que vivemos e alteramos, como agentes antrópicos. Assim, poderiam ser desenvolvidos temas relacionados aos recursos naturais, constituintes dos solos e sua preservação, ciclos biogeoquímicos e outros assuntos que oferecem integração com as demais disciplinas da educação básica. Soares, nesse trabalho que acabou endossado pela Sociedade Brasileira de Geologia como sua posição oficial perante a proposta de BNCC, enfatiza a necessidade de atividades lúdicas e interativas, como brincadeiras e jogos educativos, que explorem a compreensão dos processos naturais e sociais.

\section{Cursos de Extensão na Unicamp}

Conforme já citado, para que o professor tenha sucesso em suas aulas, é primordial possuir conhecimento científico sobre os temas que desenvolve com seus alunos. Havendo formação insuficiente em determinada área de conhecimento, ele deve recorrer a estudos complementares que o habilitem ou o aprimorem para aprofundar com seus alunos os temas da disciplina de sua responsabilidade.

Assim, o curso em questão seria organizado a partir dos temas citados na unidade temática Terra e Universo, das Ciências da Natureza, e desenvolvido no próprio IG, com a colaboração de professores e alunos dos programas de pós-graduação do Instituto.

Inicialmente, o público alvo poderia ser formado por professores da rede oficial da Prefeitura Municipal de Campinas (PMC), mediante tratativas com a sua Secretaria Municipal de Educação (SME), que realizaria gestões junto ao seu quadro docente dos anos iniciais do ensino fundamental e de Ciências da Natureza, dos anos finais também do ensino fundamental, a fim de oferecer informações quantitativas e outras complementares e peculiares daquela pasta pública, como, por exemplo, a quantidade de profissionais atuando nessas áreas da educação e seus interesses em cursos de aperfeiçoamento, para subsidiar a organização desta proposta de extensão universitária.

De acordo com a Deliberação CONSU-A-002/1999, de 07/04/1999 (Unicamp 1999), e suas alterações posteriores (Unicamp 2005), que dá nova redação ao Regulamento Interno da Extecamp, curso de extensão significa toda atividade de ensino acadêmico, técnico, cultural ou artístico, não capitulada no âmbito regulamentar de ensino de graduação e da pós-graduação "stricto sensu" da Unicamp. A mesma norma define que cursos de extensão devem possuir carga horária total mínima de 30 horas-aula. Já os cursos de atualização universitária, que são destinados a graduados em cursos superiores, têm por objetivo atualizar e melhorar conhecimentos e técnicas de trabalho e terão uma 
carga mínima de 180 horas-aula. Por sua vez, os cursos de especialização e de aperfeiçoamento, a qualquer título, destinados a graduados de cursos superiores, têm diferentes objetivos: os primeiros visam a preparar especialistas em setores restritos das atividades acadêmicas e profissionais. Os últimos buscam atualizar e melhorar conhecimentos e técnicas de trabalho. Os cursos de especialização devem ter carga horária mínima de 360 horas-aula e os de aperfeiçoamento, no mínimo, 180 horas-aula. Por fim, cursos de especialização técnica são destinados a graduados de cursos técnicos do segundo grau ou nível médio e têm por objetivo preparar especialistas em setores restritos das atividades profissionais, com carga mínima de 360 horas-aula.

A BNCC apresenta amplos e diversificados temas de Ciências da Terra em todos os anos do ensino fundamental, envolvendo áreas da Geofísica, Geologia, Meteorologia, Astronomia e outras áreas afins, de acordo com as abrangências e complexidades dessa fase da educação básica. $\mathrm{Na}$ formação/atualização docente identifica-se a necessidade de aulas teóricas, práticas de laboratórios e visitas técnicas de campo. Assim, optou-se pela organização do curso na modalidade de Aperfeiçoamento, com carga horária mínima de 180 horas-aula, a ser desenvolvido em um semestre letivo.

\subsection{Plataforma Moodle}

Atualmente, a Extecamp utiliza a plataforma Moodle para cursos à distância ou semipresenciais. A plataforma Moodle (Modular Object Oriented Distance Learning) é um sistema destinado ao gerenciamento de curso online, que oferece os Ambientes Virtuais de Aprendizagem (AVA) ou Learning Management System (LMS). Trata-se de software livre de apoio à aprendizagem, comumente utilizado para o ensino à distância, com ou sem fase presencial.

Ao oferecer recursos de aprendizado online, esse sistema substitui materiais físicos, como cópias e apostilas impressas, economizando recursos e agilizando o acesso a tais materiais.

\subsection{Público Alvo}

A opção pelos professores formados em Pedagogia, que lecionam nos anos iniciais do ensino fundamental, e de Ciências da Natureza dos anos finais desse mesmo ciclo de ensino, ocorreu em razão de serem esses docentes os responsáveis por desenvolver temas de Ciências da Terra nessa primeira fase da educação básica. A nova BNCC destina a esses professores o ensino de áreas do conhecimento envolvendo Terra e Universo, dentre outras.

Consta no edital do concurso mais recente da SME do município de Campinas, realizado no ano de 2016 (PMC 2016), a admissão de professores denominados Adjuntos I, para atuarem nos anos iniciais do ensino fundamental, com formação em cursos de Licenciatura Plena em Pedagogia ou Normal Superior, ambos com habilitação específica.

Para atuarem na disciplina de Ciências da Natureza dos anos finais do ensino fundamental, a destinação do cargo de professor dessa disciplina ocorre aos candidatos possuidores de Licenciatura Plena em Ciências da Natureza, ou Ciências Físicas e Biológicas, ou História Natural, ou Licenciatura Plena em Ciências com habilitação específica em Física ou Biologia ou Química. Estudos sobre esses cursos de formação de professores poderão indicar se as suas grades curriculares contemplam, em geral, disciplinas que tratam das Ciências da Terra com a mesma profundidade apresentada na atual proposta da BNCC.

\subsection{Justificativa}

Cursos de extensão têm por objetivo atualizar e melhorar conhecimentos e técnicas de trabalho, preparar especialistas em setores restritos das atividades acadêmicas e profissionais, ampliando a efetividade da construção de conhecimentos, disponibilizando para a comunidade os recursos educacionais existentes na universidade. Trata-se de um dos pilares de sustentação da universidade, associado ao ensino e à pesquisa.

Por sua vez, em razão da evolução da educação, professores devem se preparar para novas práticas e atualização de seus conhecimentos e de suas formas de trabalhar estes conhecimentos com os alunos. Assim, a realização de curso de extensão para professores de Ciências da Natureza justifica-se ao proporcionar a atualização de docentes da educação básica, em particular, valorizando as Ciências da Terra nessa fase do aprendizado e proporcionando aos professores recursos para tratarem esses temas a partir de uma visão holística ou integrada.

Para Krasilchik \& Marandino (2004), o estudo das Ciências da Natureza deve provocar nos estudantes, e também na população em geral, a curiosidade, e levá-los a se dar conta do papel que esses conhecimentos têm em suas vidas, especialmente para o pleno exercício da cidadania. Por sua vez,

\begin{tabular}{c|c|c|c|c|c}
\hline (C) Terrae Didat. & Campinas, SP & v.15 & $1-8$ & e019021 & 2019 \\
\hline
\end{tabular}


Bizzo (1998) afirma que o trabalho do professor de Ciências da Natureza deve ser pautado pelo sucesso do aluno.

A mais recente versão da BNCC para o ensino fundamental (Brasil 2017) apresenta diversificadas áreas de conhecimentos na unidade temática Terra e Universo, da área das Ciências da Natureza. Contudo, Piranha \& Carneiro (2009) consideram que em alguns casos, grande parte dos professores da educação básica reconhece a necessidade de aprofundar seus conhecimentos em Ciências da Terra, porque sabem que tiveram uma formação insuficiente para o exercício pleno e seguro das suas atividades docentes nessa área. A deficiência pode ocorrer porque grande parte dos professores de Ciências da Natureza é constituída por docentes formados em Biologia (Waldhelm 2007).

Araujo \& Toledo (2014) observam que os Parâmetros Curriculares Nacionais (PCN), suportes da BNCC, já apresentavam a necessidade de serem trabalhados com os alunos da educação básica temas de Astronomia, Meteorologia, Geologia e Geofísica, dentre outros, não oferecidos na formação básica de professores de Ciências Naturais. Em estudos de caso desenvolvidos em instituições de ensino superior, os autores verificaram que os componentes curriculares da formação de professores apresentam cargas horárias insuficientes em Ciências da Terra, fato que pode conduzir à formação insatisfatória dos egressos de determinados cursos de licenciatura, correspondentes a cursos de Biologia que habilitam ao magistério de Ciências Naturais.

Da mesma forma, existem ponderações sobre o biólogo lecionar Ciências da Natureza. Cunha \& Krasilchik (2000) afirmam que as licenciaturas em Ciências Biológicas, estejam elas vinculadas ou não a cursos de bacharelado, estão longe de formar adequadamente o professor de Ciências da Natureza para o ensino fundamental. Para as autoras, os currículos dos cursos de Ciências Biológicas são altamente "biologizados".

No Brasil, além de a maioria dos atuais professores de Ciências da Natureza ser formada em curso de Pedagogia ou Ciências Biológicas, habilitados para lecionar Ciências, e, assim, possivelmente não possuir suficientes conhecimentos e preparo em temas de Ciências da Terra, os assuntos estariam dispersos no currículo da educação básica; a falta dessa ordenação seria uma das condições responsáveis pela precariedade do ensino e da compreensão da dinâmica do planeta (Carneiro et al. 2004).
Na mesma linha de pensamento, Libâneo \& Pimenta (2006) afirmam que a formação inicial seria insuficiente para formar plenamente o professor e, por essa razão, sinalizam sobre a importância do investimento no desenvolvimento profissional, que pode ser definido como a formação continuada de professores, por meio de cursos de aperfeiçoamento.

\subsection{Proposta inicial para a estrutura do curso}

Conforme já citado, o curso de extensão será desenvolvido como aperfeiçoamento, na modalidade semipresencial, composto pela aula zero, seis disciplinas e as atividades práticas de campo.

Será destinado a professores dos anos iniciais e anos finais do ensino fundamental, respectivamente, Pedagogos que atuam do $1^{\circ}$ ao $5^{\circ}$ ano e professores de Ciências da Natureza do $6^{\circ}$ ao $9^{\circ}$ ano. Em razão de esses professores possuírem formações distintas e lecionarem em ciclos diferentes do ensino fundamental, serão organizados em turmas diferentes. Contudo, poderão ser agrupados em única turma, casos estudos indiquem essa possibilidade ou necessidade.

Para contemplar os conteúdos descritos na unidade temática Terra e Universo da BNCC, os assuntos serão organizados em seis disciplinas: (a) Astronomia para Ciências da Natureza; (b) Meteorologia para Ciências da Natureza; (c) Sistema Terra: Dinâmica Interna; (d) Sistema Terra: Dinâmica Externa; (e) Minerais e Ciclo das Rochas; e (f) Geologia Ambiental e Tempo Geológico, além da Atividade Prática e Interdisciplinar de Campo que encerra o curso.

A Tabela 1 apresenta o resumo da distribuição das atividades e suas cargas horárias.

A Aula Zero destina-se à recepção dos grupos de discentes para a apresentação do curso, da coordenação, professores e disciplinas, do acesso e operação do AVA, dos materiais didáticos, das dinâmicas das atividades online e presenciais, das avaliações de aprendizagem, das atividades práticas de campo e demais peculiaridades do curso. Os alunos serão cientificados sobre a condição de se tratar de um curso desenvolvido como projeto-piloto e, por essa razão, além dos ajustes que poderão ocorrer durante seu desenvolvimento, haverá pesquisas para verificar a percepção dos grupos envolvidos e a evolução do curso.

Nas aulas subsequentes serão oferecidas as seis disciplinas, cada uma com duração de 30 horas- 
Tabela 1. Atividades previstas e cargas horárias do curso de aperfeiçoamento para professores de Ciências da Natureza

Aperfeiçoamento para Professores de Ciências da Natureza

\begin{tabular}{l|l|l|l}
\hline \multirow{2}{*}{ Conteúdos programáticos ou disciplinas } & \multicolumn{2}{|c}{ Cargas Horárias } & Totais \\
\cline { 2 - 4 } & $\mathbf{A O}^{\star}$ & \multicolumn{1}{c}{$\mathbf{A P}^{\star \star}$} & 4 \\
\hline Aula Zero: apresentações & 20 & 10 & 30 \\
\hline Astronomia para Ciências da Natureza & 20 & 10 & 30 \\
\hline Meteorologia para Ciências da Natureza & 20 & 10 & 30 \\
\hline Sistema Terra: Dinâmica Interna & 20 & 10 & 30 \\
\hline Sistema Terra: Dinâmica Externa & 20 & 10 & 30 \\
\hline Minerais e Ciclo das Rochas & 20 & 10 & 30 \\
\hline Geologia Ambiental e Tempo Geológico & 4 & 8 & 12 \\
\hline Atividade Prática e Interdisciplinar de Campo & 124 & 72 & $\mathbf{1 9 6}$ \\
\hline & \multirow{2}{*}{ AO: atividades online. **AP: atividades presenciais. }
\end{tabular}

-aula distribuídas em cinco semanas, sendo quatro horas-aula de atividades online e duas horas-aula de atividades presenciais, aos sábados. Para que o curso seja desenvolvido em apenas um semestre letivo, essas seis disciplinas serão oferecidas de duas em duas. Dessa forma, as atividades online semanais corresponderão a oito horas-aula e as atividades presenciais aos sábados a quatro horas-aula.

Nos encontros ou aulas presenciais serão discutidas as atividades online trabalhadas durante a semana, realizadas trocas de experiências, promovidas algumas dinâmicas e realizadas as avaliações de aprendizagem.

Para finalizar o curso, serão desenvolvidas atividades de campo, com quatro horas-aula de atividades online e o campo em si, no último sábado letivo, com oito horas-aula de trabalhos práticos de campo científico-pedagógicos, totalizando as 12 horas-aula propostas.

\section{Considerações Finais}

O desenvolvimento adequado do tema "Terra e Universo", previsto pela BNCC do ensino fundamental constitui um enorme desafio para docentes graduados em cursos de Pedagogia, que habilitam professores para atuar nos anos iniciais do ensino fundamental ou cursos de Licenciatura em Biologia, que estão normalmente incumbidos das aulas de Ciências da Natureza nos anos finais do mesmo ensino fundamental. A principal razão de a tarefa constituir um desafio é o preparo insuficiente e a pequena carga horária presente nas respectivas grades curriculares de disciplinas de Geologia e/ ou Paleontologia em seus cursos de formação. Em muitos casos, a carga horária é pequena, limitada a apenas uma disciplina para formar alunos nessas áreas do conhecimento científico.

A proposta, descrita neste artigo, de curso de extensão universitária nas áreas de conhecimento da unidade temática Terra e Universo, como projeto-piloto, procura valorizar as Ciências da Terra e minimizar os efeitos da situação acima referida. A experiência dos autores em atividades de extensão sugere ser grande a chance de êxito desse tipo de curso de extensão para professores de Ciências da Natureza.

\section{Agradecimentos}

Os autores agradecem ao Conselho Nacional de Desenvolvimento Científico e Tecnológico $\mathrm{CNPq}$ - pela concessão de bolsa de Produtividade em Pesquisa a um dos autores (CDRC).

\section{Referências}

Araujo, E. P. R. de; \& Toledo, M. C. M. de. (2014). Ciências da Terra em cursos que habilitam ao magistério de Ciências Naturais para o ensino fundamental. Terræ Didatica, 10(3), p. 319-330. doi: 10.25249/0375-7536.2004344553560.

Bizzo, N. (2009). Mais Ciências no ensino fundamental: metodologia de ensino em foco. São Paulo: Ed. Brasil.

Brasil. (1988). Constituição da República Federativa do Brasil. Brasília: Casa Civil. URL: http://www.planalto.gov.br/ccivil_03/constituicao/constituicao.htm. Acesso 26.01.2018.

Brasil. (1996). Lei Federal no 9.394, de 20/12/1996. Estabelece as diretrizes e bases da educação nacional (LDB). Brasília: Casa Civil. URL: http://www.planalto.gov. br/ccivil_03/Leis/L9394.htm. Acesso 26.01.2018.

Brasil. (2014). Lei Federal no 13.005, de 25/07/2014. Aprova o Plano Nacional de Educação (PNE). 
Brasília: Casa Civil. URL: http://www.planalto. gov.br/ccivil_03/_ato2011-2014/2014/lei/113005. htm. Acesso 26.01.2018.

Brasil. (2017). Base Nacional Comum Curricular para o Ensino Fundamental (BNCC). Brasília: Ministério da Educação. URL: http://basenacionalcomum.mec.gov.br/images/BNCCpublicacao.pdf. Acesso 28.12.2017.

Carneiro, C. D. R.; Toledo, M. C. M. de; \& Almeida, F. F. M. de. (2004). Dez motivos para a inclusão de temas de Geologia na Educação Básica. Rev. Bras. Geoc., 34(4), p. 553-560. URL: sbgeo.org.br/ pub_sbg/rbg/vol34_down/3404/1439.PDF. Acesso 18.09.2017.

Cunha, A. M. O.; \& Krasilchik M. (2000). A formação continuada de professores de Ciências: percepções a partir de uma experiência. In: Reunião Assoc. Nac. Pós-Grad. Pesq. Educação, 23, Caxambu. Anais... Caxambu: ANPGPE.

Krasilchik, M.; \& Marandino, M. (2004). Ensino de ciências e cidadania. São Paulo: Moderna.

Libâneo, C. J.; \& Pimenta, S. G. (2006). Formação dos profissionais da Educação: visão crítica e perspectivas de mudança. In: Pimenta S.G. org. (2006). Pedagogia e Pedagogos: caminhos e perspectivas. São Paulo: Cortez.

Piranha, J. M.; \& Carneiro, C. D. R. (2009). O módulo São José do Rio Preto do Projeto Geo-Escola, uma experiência educacional diferenciada. Rev.
Bras. Geoc., 39(3), p. 533-543. URL: http://ppegeo. igc.usp.br/index.php/rbg/article/view/7696/7123. Acesso 12.12.2017.

Prefeitura Municipal de Campinas. PMC. (2016). Edital $n^{\circ} 03$, de 21/03/2016. Concurso Público para provimento de cargos públicos da área da Educação. Diário Oficial de Campinas no 11.321, ano XLV, de 24/03/2016, p. 26-37. Campinas: Secretaria Municipal de Recursos Humanos. URL: http:// campinas.sp.gov.br/uploads/pdf/1436064064.pdf. Acesso 31.01.2018.

Soares, P. C. (2016). As Ciências da Terra nas Ciências da Natureza nas diretrizes curriculares do ensino básico, no Brasil. Curitiba, SBG. (documento endossado pela Soc. Bras. Geologia e encaminhado ao MEC, inédito).

Universidade Estadual de Campinas (Unicamp). (1999). Deliberação CONSU-A-002/1999, de 07/04/1999. Campinas, Unicamp. URL: http:// www.pg.unicamp.br/mostra_norma.php?id_nor$\mathrm{ma}=2636$. Acesso 30.01.2018.

Universidade Estadual de Campinas (Unicamp). (2005). Deliberação CONSU-A-009/2005, de 01/06/2005. Campinas, Unicamp. URL: http:// www.pg.unicamp.br/mostra_norma.php?id_nor$\mathrm{ma}=2814$. Acesso 30.01.2018.

Waldhelm, M. C. V. (2007). Como aprendeu ciências na educação básica quem hoje produz ciência? Tese de Doutorado. Rio de Janeiro, PUC/RJ. 